\title{
Uso de substâncias psicoativas entre universitários de medicina da Universidade Federal do Espírito Santo
}

\author{
Psychoactive substance use among Medicine students \\ from Espirito Santo Federal University
}

Denis Soprani Pereira', Renata Santos de Souza', Vitor Buaiz², Marluce Miguel de Siqueira²

\section{RESUMO}

Objetivo: Traçar o perfil do uso de substâncias psicoativas entre os universitários do Curso de Medicina do Centro de Ciências da Saúde da Universidade Federal do Espírito Santo. Métodos: Trata-se de um estudo exploratório, descritivo, transversal e quantitativo, desenvolvido com 168 universitários, do primeiro ao último ano do curso de medicina. O instrumento utilizado na coleta de dados foi o Questionário sobre o Uso de Drogas, uma adaptação do questionário proposto pela OMS'. Os dados foram tabulados por meio do programa Statistical Package for the Social Science (SPSS) ${ }^{2}$. Resultados: Ao todo, 54,8\% dos universitários são do sexo feminino, 76,8\% se encontram na faixa etária de 17 a 22 anos e $50 \%$ pertencem à classe social B. Quanto ao uso de substâncias psicoativas, 86,9\% relataram uso na vida de álcool, seguido de tabaco (22,0\%), solventes (15,5\%), anfetaminas (10,1\%), cannabis sativa (9,5\%), alucinógenos com 1,8\% e barbitúricos com 0,6\%. Conclusão: Faz-se necessária a prevenção do uso indevido de substâncias psicoativas entre universitários, por meio de disciplinas curriculares que abordem a temática e de programas de prevenção destinados a essa população.

\section{ABSTRACT}

Objective: To describe the profile of the psychoactive substances used among the college students from the Medicine course in the Center of Health Sciences in, Espirito Santo Federal University. Methods: It is a exploratory, descriptive, transversal and quantitative study developed within 168 college students of Medicine from the first to the last year of the course. The instrument used to collect data was the Questionnaire on Drug Abuse, an adaptation from the questionnaire proposed by WHO'. The data had been tabulated through the Social Science Program Statistical Package (SPSS)'. Results: Overall, 54.8\% of the college students are females, 76.8\%, from 17 to 22 years and $50 \%$ belonging to social class " $B$ ". As for the use of psychoactive substances, a high prevalence of alcohol was founded (86.9\%), followed by tobacco (22.0\%), solvents (15.5\%), amphetamines (10.1\%), cannabis (9.5\%), hallucinogens (1.8\%) and barbiturates (0.6\%).

\section{Keywords}

Psychoactive substances, colleges, prevention.

Conclusion: It is necessary to prevent the psychoative substance abuse among college students, introducing curricular disciplines on the theme or specific programs to attend these students.

1 Departamento de Enfermagem do Núcleo de Estudos sobre Álcool e outras Drogas da Universidade Federal do Espírito Santo (NEAD-UFES). 2 Departamento de Clínica Médica Núcleo de Estudos sobre Álcool e outras Drogas da Universidade Federal do Espírito Santo (NEAD-UFES). 
O crescente consumo de substâncias psicoativas (SPAs) é uma problemática que acarreta conseqüências devastadoras à sociedade, destacando-se seus impactos sociais e econômicos, que o caracteriza como importante problema de saúde pública, principalmente entre os jovens ${ }^{3}$. Tal fato, associado à escassez de dados que contemplem esta questão, motiva pesquisas que surgem por todo o Brasil e que são indispensáveis para fazer valer um dos pressupostos da Política Nacional sobre Drogas ${ }^{4}$ - que é obter uma sociedade livre do uso de SPAs (lícitas e ilícitas) ilícitas e do uso indevido de SPAs lícitas.

Conhecer o padrão do consumo de substâncias psicoativas de determinada população é essencial à implantação de programas de prevenção ao consumo de drogas, pois, "ter informações acerca dos padrões de consumo, além de eliminar mitos existentes sobre o assunto, possibilita um direcionamento para o desenvolvimento de políticas públicas, incluindo prevenção e tratamento, com resultados potencialmente mais proveitosos"3.

Na população brasileira, o uso na vida de SPAs ilícitas abrange 22,8\% da população, enquanto 44\% e 74,6\% já fizeram uso na vida de tabaco e álcool, respectivamente e, ainda 12,3\% (5.799.005 de pessoas) são dependentes do álcool ${ }^{5}$.

A realização deste estudo com os universitários do curso de medicina da Universidade Federal do Espírito Santo (UFES) se faz relevante, haja vista que estes estudantes, futuros profissionais da saúde, serão os responsáveis pelo diagnóstico, pelas intervenções e pelos encaminhamentos de pacientes com as mais diversas doenças, inclusive aquelas relacionadas ao uso de SPAs. Deve-se acrescer, ainda, que o profissional médico deve ter cuidado com sua conduta, uma vez que o paciente o toma como modelo a seguir e que este profissional tem o agravante de se situar em um grupo de risco para abuso destas SPAs, pois seu ambiente de trabalho proporciona fácil acesso a elas, concomitante à fadiga e ao estresse inerentes à profissão ${ }^{6}$.

Segundo Souza, Landim e Perdigão7, o consumo de drogas começa a causar seus impactos já na vida acadêmica, resultando falta de atenção durante as aulas, ausências, atrasos e maior sonolência. Os estudos de Boskovitz, Cruz e Chiaravalotti-Neto ${ }^{8}$ e Galduróz et al. ${ }^{9}$ corroboram com este achado.

Existe ainda aumento da prevalência do consumo de substâncias psicoativas com o passar dos anos letivos no curso de medicina, visto que no primeiro ano encontrou-se prevalência de 16,1\% e no quinto ano 43,7\%,70.

A assistência à saúde de determinada população depende, muitas vezes, da integridade física e mental do médico. Assim, a falta desse tema no currículo acadêmico, além de potencializar risco à saúde da população, pelo envolvimento com drogas desses universitários de medicina, pode influenciar no serviço prestado ao fármaco-dependente.
Segundo Kerr-Correa et al. ${ }^{10}$, mais de 50\% dos estudantes de medicina da UNESP, não acreditam na recuperação do paciente alcoolista.

Portanto, o objetivo deste estudo foi detectar o perfil dos usuários de substâncias psicoativas entre os universitários do curso de medicina da UFES. Este perfil fornecerá subsídios para implantação de um futuro programa de prevenção ao uso indevido de substâncias psicoativas dirigido a estes universitários.

\section{MÉTODOS}

A pesquisa foi realizada no Curso de Medicina do Centro de Ciências da Saúde (CCS) da UFES, durante o mês de novembro de 2007, sendo o estudo de desenho transversal, quantitativo, exploratório e descritivo ${ }^{11,12}$

A população do estudo foi constituída de estudantes matriculados no referido curso nos 12 períodos acadêmicos, totalizando 501 alunos. A amostra foi calculada no software Epi Info 6.04, considerando-se nível de significância de 5\%, precisão de $5 \%$ e prevalência esperada de uso na vida de álcool de $80 \%$, com base nos resultados dos estudos sobre o uso de substâncias psicoativas de Andrade et al.13, Mesquita, Bucaretchi e Caste ${ }^{14}$ e Kerr-Correa et al. ${ }^{10}$, obtendo-se amostra de 166 estudantes. Entretanto, como a coleta de dados foi realizada com todos os alunos que estavam presentes em sala de aula no dia da aplicação do instrumento e que aceitaram participar da pesquisa, a amostra final foi constituída de 168 estudantes.

Anteriormente à coleta de dados, foi realizado um estudo-piloto com alunos do início, do meio e do final do curso de medicina, no qual se verificou que a questão 36, necessitava de retificação, o que foi feito no presente estudo.

A pesquisa foi aprovada pelo Comitê de Ética em Pesquisa do CCS da UFES, por meio do Processo no 104/2006. Após sua aprovação, foi enviada uma carta ao coordenador do colegiado do curso de medicina, solicitando a colaboração dos professores, disponibilizando alguns minutos de suas aulas para a aplicação dos questionários.

Foi utilizado um questionário auto-preenchível com questões fechadas e semi-abertas de caráter anônimo, sendo este preconizado pela Organização Mundial da Saúde (OMS) para pesquisas acerca do uso de drogas entre estudantes? O instrumento denominado Questionário sobre o Uso de Drogas, traduzido e adaptado à realidade brasileira por Carlini-Cotrim ${ }^{15}$, foi aplicado por uma equipe previamente treinada de bolsistas de iniciação científica, com a prévia autorização do professor. Aqueles estudantes que aceitaram participar da pesquisa assinaram o Termo de Consentimento Livre e Esclarecido, de acordo com a Resolução no 196/1996, do Conselho Nacional de Saúde ${ }^{16}$. 
O questionário utilizado no estudo é composto de cinco partes. A primeira parte apresenta a pesquisa e convida o estudante a participar dela. A segunda é composta por questões a respeito do uso de SPAs (álcool, tabaco, maconha, alucinógenos, cocaína, anfetaminas, anticolinérgicos, solventes orgânicos, tranqüilizantes ansiolíticos, opiáceos, sedativos e barbitúricos). A terceira compreende questões socioeconômicas que foram avaliadas segundo a escala proposta pela Associação Brasileira dos Institutos de Pesquisa e Mercado ${ }^{17}$. A quarta está formada por questões que visam a detalhar alguns aspectos do consumo do álcool; e a quinta é composta por questões acerca do relacionamento intrafamiliar. É importante salientar que o questionário utilizado mede o relato da freqüência de uso de SPAs e não seu uso propriamente dito. Tal fato, de maneira alguma, torna menos valiosos os dados obtidos no tocante ao fomento de políticas públicas sobre álcool e outras drogas, com destaque para a prevenção ao uso ${ }^{18}$.

Os dados foram tabulados por meio do programa Statistical Package for the Social Science (SPSS)', seguindose a análise descritiva das variáveis. Os dados referentes ao consumo de drogas pelos universitários foram analisados de acordo com os indicadores de classificação sobre o uso de drogas, preconizados pela OMS': uso na vida (quando a pessoa fez uso pelo menos uma vez na vida); uso no ano (quando a pessoa fez uso pelo menos uma vez nos 12 meses que antecederam a pesquisa); uso no mês (quando a pessoa fez uso pelo menos uma vez nos 30 dias que antecederam a pesquisa); uso freqüente (quando a pessoa fez uso seis ou mais vezes nos 30 dias que antecederam a pesquisa); e uso pesado (quando a pessoa fez uso 20 ou mais vezes nos 30 dias que antecederam a pesquisa).

Esta pesquisa foi aprovada pelos comitês de ética da Universidade Federal do Espírito Santo, e todos os indivíduos assinaram termo de consentimento e esclarecido antes de qualquer procedimento do estudo fosse iniciado.

\section{RESULTADOS}

Em relação aos dados socioeconômicos dos universitários, pode-se observar na Tabela 1 que, em sua maioria, estes estudantes são do sexo feminino (54,8\%), concentrados na faixa etária de 17 a 22 anos (76,8\%) e, segundo a Abipeme ${ }^{17}$, nas classes socioeconômicas B $(50,0 \%)$ e A $(40,5 \%)$. Os critérios que regem a estratificação dos sujeitos em classes socioeconômicas, definidos pela ABIPEME, levam em consideração itens de conforto e nível de escolaridade, atribuindo, então, pontos a estes, o que permite diferenciar os estudantes em diferentes classes socioeconômicas.
Tabela 1. Características socioeconômicas dos estudantes do curso de medicina do Centro de Ciências da Saúde da Universidade Federal do Espírito Santo, Vitória, 2007.

\begin{tabular}{lcc}
\hline \multicolumn{1}{c}{ Caracteristicas } & $\mathbf{n}$ & $\%$ \\
\hline Sexo & 74 & \\
$\quad$ Masculino & 92 & 44,0 \\
Feminino & 2 & 54,8 \\
Não informado & 168 & 1,2 \\
$\quad$ Total & & 100 \\
Faixa etária (anos) & 35 & \\
17 a 19 & 94 & 20,8 \\
20 a 22 & 94 & 56,0 \\
23 a 25 & 29 & 17,3 \\
26 a 28 & 4 & 2,4 \\
$\geq 29$ & 3 & 1,8 \\
Não informado & 3 & 1,8 \\
Total & 168 & 100 \\
Faltas à Universidade & & \\
Nenhuma falta & 47 & 28,0 \\
1 a 3 faltas & 60 & 35,7 \\
4 a 8 faltas & 28 & 16,7 \\
9 ou mais faltas & 32 & 19,0 \\
Não informado & 1 & 0,6 \\
Total & 168 & 100 \\
Nivel socioeconômico & & \\
A & 68 & 40,5 \\
B & 84 & 50,0 \\
C & 15 & 8,9 \\
D & 1 & 0,6 \\
E & 0 & 0 \\
Não informado & 0 & 0 \\
Total & 168 & 100 \\
\hline
\end{tabular}

Pode-se visualizar, também, que estes estudantes têm taxa elevada de faltas à universidade, porquanto 35,7\% disseram que faltaram de uma a três vezes nos últimos 30 dias e 19\% disseram ter faltado nove vezes ou mais.

Em relação ao uso de substâncias psicoativas (Tabela 2), observa-se que o álcool e o tabaco são as substâncias de maiores prevalências de uso na vida, com 86,9\% e, 22\%, respectivamente. Quanto às demais substâncias, na categoria uso na vida, figuram entre as mais preocupantes os solventes (15,5\%), os ansiolíticos (13,1\%), os anfetamínicos $(10,1 \%)$ e a cannabis sativa $(9,5 \%)$. Ao se analisar o uso no mês das substâncias psicoativas, pode-se observar o consumo de álcool (48,2\%), anfetamínicos (3,0\%), ansiolíticos $(2,4 \%)$ e tabaco $(1,2 \%)$. Apenas o álcool $(3,7 \%)$ e o tabaco $(1,8 \%)$ apresentaram uso freqüente entre os estudantes. E, em relação ao uso pesado, foram encontrados relatos de uso de álcool (3,0\%), ansiolíticos (1,8\%), anfetamínicos (1,2\%), tabaco $(0,6 \%)$ e solventes $(0,6 \%)$.

Na Tabela 3, pode-se observar que o consumo de álcool pouco difere em relação ao sexo dos estudantes, visto que $89,2 \%$ dos homens já usaram álcool e 84,8\% das mulheres também o fizeram. Quanto ao consumo de tabaco $(25,7 \%)$, cannabis sativa $(13,5 \%)$ e solventes (18,9\%), os homens possuem taxas significantemente maiores em relação às mulheres, entretanto, quanto ao uso de ansiolíticos (16,3\%), barbitúricos (1,1\%) e alucinógenos (2,2\%), a prevalência é maior no sexo feminino. 
Tabela 2. Uso de substâncias psicoativas (na vida, no ano, no mês, freqüente e pesado) entre estudantes do curso de medicina do Centro de Ciências da Saúde da Universidade Federal do Espírito Santo, Vitória, 2007.

\begin{tabular}{|c|c|c|c|c|c|c|c|c|c|c|}
\hline \multirow{2}{*}{ Drogas } & \multicolumn{2}{|c|}{ Na vida } & \multicolumn{2}{|c|}{ No ano } & \multicolumn{2}{|c|}{ Nomês } & \multicolumn{2}{|c|}{ Freqüente } & \multicolumn{2}{|c|}{ Pesado } \\
\hline & $\mathrm{n}$ & $\%$ & $n$ & $\%$ & $\mathrm{n}$ & $\%$ & $n$ & $\%$ & $\mathrm{n}$ & $\%$ \\
\hline Ansiolíticos & 22 & 13,1 & 15 & 8,9 & 4 & 2,4 & & 0 & 3 & 1,8 \\
\hline Anfetamínicos & 17 & 10,1 & 14 & 8,3 & 5 & 3,0 & & 0 & 2 & 1,2 \\
\hline Solventes & 26 & 15,5 & 10 & 6,0 & 1 & 0,6 & & 0 & 1 & 0,6 \\
\hline Maconha & 16 & 9,5 & 7 & 4,2 & 0 & 0 & 0 & 0 & 0 & 0 \\
\hline Barbitúricos & 1 & 0,6 & 0 & 0 & 0 & 0 & 0 & 0 & 0 & 0 \\
\hline Anticolinérgicos & 0 & 0 & 0 & 0 & 0 & 0 & 0 & 0 & 0 & 0 \\
\hline Cocaína & 0 & 0 & 0 & 0 & 0 & 0 & 0 & 0 & 0 & 0 \\
\hline Alucinógenos & 3 & 1,8 & - & - & - & - & - & - & - & - \\
\hline Opiáceos & 0 & 1,2 & - & - & - & - & - & - & - & - \\
\hline Xaropes & 1 & 0,6 & - & - & - & - & - & - & - & - \\
\hline Orexígenos & 0 & 0 & - & - & - & - & - & - & - & - \\
\hline Total de usuários & \multicolumn{2}{|c|}{50,4} & \multicolumn{2}{|c|}{27,4} & \multicolumn{2}{|c|}{6,0} & \multicolumn{2}{|c|}{0} & \multicolumn{2}{|c|}{3,6} \\
\hline Álcool & 146 & 86,9 & 138 & 82,1 & 81 & 48,2 & 23 & 13,7 & 5 & 3,0 \\
\hline Tabaco & 37 & 22,0 & 18 & 10,7 & 2 & 1,2 & 3 & 1,8 & 1 & 0,6 \\
\hline
\end{tabular}

Tabela 3. Uso na vida de substâncias psicoativas entre os estudantes do curso de medicina do Centro de Ciências da Saúde da Universidade Federal do Espírito Santo - distribuição por sexo, Vitória, 2007.

\begin{tabular}{|c|c|c|c|c|c|}
\hline \multirow{2}{*}{ Droga } & \multicolumn{2}{|c|}{ Masculino } & \multicolumn{2}{|c|}{ Feminino } & \multirow{2}{*}{$\begin{array}{c}\text { Total } \\
\mathbf{n} \\
\end{array}$} \\
\hline & $n$ & $\%$ & $\mathrm{n}$ & $\%$ & \\
\hline Álcool & 68 & 89,2 & 78 & 84,8 & 146 \\
\hline Tabaco & 19 & 25,7 & 18 & 19,6 & 37 \\
\hline Maconha & 10 & 13,5 & 6 & 6,5 & 16 \\
\hline Cocaína & 0 & 0 & 0 & 0 & 0 \\
\hline Anfetamínicos & 8 & 10,8 & 9 & 9,8 & 17 \\
\hline Solventes & 14 & 18,9 & 12 & 13,0 & 26 \\
\hline Ansiolíticos & 7 & 9,5 & 15 & 16,3 & 22 \\
\hline Anticolinérgicos & 0 & 0 & 0 & 0 & 0 \\
\hline Barbitúricos & 0 & 0 & 1 & 1,1 & 1 \\
\hline Alucinógenos & 1 & 1,4 & 2 & 2,2 & 3 \\
\hline
\end{tabular}

A idade de experimentação das drogas lícitas pesquisadas (Tabela 4) correspondeu à faixa etária compreendida entre 13 e 18 anos para o álcool e de 16 a 18 anos para o tabaco. No entanto, houve percentual considerável de experimentação abaixo dos 12 anos (8,9\%) para o álcool. Entre as drogas ilícitas, a idade de experimentação para os solventes, cannabis sativa, anfetamínicos e ansiolíticos foi de 16 a 18 anos. Entretanto, boa parte dos estudantes experimentou solventes entre 13 e 15 anos de idade.

Em relação ao consumo de bebidas alcoólicas, pode-se perceber (Tabela 5) que as bebidas preferidas pelos estudantes foram: a cerveja $(50,4 \%)$ e a vodca $(15,1 \%)$. Segundo os universitários, ao sair para beber, 44,6\% bebem de 1 a 2 doses, 21,9\% bebem de 3 a 4 doses e 21,9\% bebem mais que 5 doses. Os bares, as danceterias e as boates são os locais preferidos para o consumo $(69,2 \%)$ e para $78,1 \%$ este consumo ocorre na presença de amigos. Quanto à freqüência de embriaguez, 17,8\% dos estudantes disseram ter se embriagado de 1 a 5 vezes no último mês.

$\mathrm{Na}$ Tabela 6 pode-se perceber uma realidade perigosa e que sugere quais seriam algumas das causas para o relevante impacto socioeconômico advindo do consumo de álcool, em que 18,5\% dos estudantes disseram já ter dirigido após ingerir bebidas alcoólicas, 14,4\% faltaram à universidade e $11,7 \%$ sofreram acidentes ou se envolveram em brigas após o uso de bebidas alcoólicas.

Tabela 4. Uso de substâncias psicoativas entre estudantes de medicina do Centro de Ciências da Saúde da Universidade Federal do Espírito Santo - idade de experimentação, Vitória, 2007.

\begin{tabular}{|c|c|c|c|c|c|c|c|c|c|c|c|c|c|}
\hline \multirow{2}{*}{ Droga } & \multicolumn{2}{|c|}{ Abaixo de 10 anos } & \multicolumn{2}{|c|}{$10-12$ anos } & \multicolumn{2}{|c|}{ 13-15 anos } & \multicolumn{2}{|c|}{ 16-18 anos } & \multicolumn{2}{|c|}{ Acima de 18 anos } & \multicolumn{2}{|c|}{ Nãolembra } & \multirow{2}{*}{ Total } \\
\hline & $n$ & $\%$ & $n$ & $\%$ & $n$ & $\%$ & $n$ & $\%$ & $n$ & $\%$ & $\mathrm{n}$ & $\%$ & \\
\hline Maconha & 0 & 0 & 0 & 0 & 1 & 6,3 & 10 & 62,5 & 5 & 31,3 & 0 & 0 & 16 \\
\hline Anfetamínicos & 0 & 0 & 1 & 5,9 & 0 & 0 & 3 & 17,6 & 13 & 76,5 & 0 & 0 & 17 \\
\hline Solventes & 3 & 11,5 & 0 & 0 & 4 & 15,4 & 9 & 34,6 & 8 & 30,8 & 0 & 0 & 25 \\
\hline Ansiolíticos & 1 & 4,5 & 0 & 0 & 0 & 0 & 6 & 27,3 & 12 & 54,5 & 3 & 13,6 & 22 \\
\hline Barbitúricos & 0 & 0 & 0 & 0 & 0 & 0 & 1 & 100 & 0 & 0 & 0 & 0 & 1 \\
\hline Tabaco & 2 & 5,4 & 1 & 2,7 & 6 & 16,2 & 16 & 43,2 & 8 & 21,6 & 4 & 10,8 & 37 \\
\hline Álcool & 2 & 1,4 & 11 & 7,5 & 41 & 28,1 & 58 & 39,7 & 8 & 11,8 & 26 & 17,8 & 146 \\
\hline
\end{tabular}


Tabela 5. Características dos estudantes do curso de medicina do Centro de Ciências da Saúde da Universidade Federal do Espírito Santo em relação ao consumo de bebidas alcoólicas, Vitória, 2007.

\begin{tabular}{|c|c|c|}
\hline \multirow{2}{*}{ Variáveis } & \multicolumn{2}{|c|}{ Uso nomês } \\
\hline & $\mathrm{n}$ & $\%$ \\
\hline \multicolumn{3}{|l|}{ Bebidas consumidas } \\
\hline Cerveja & 74 & 50,4 \\
\hline Vodca & 22 & 15,1 \\
\hline Vinho & 12 & 8,2 \\
\hline Conhaque & 1 & 0,7 \\
\hline Outros & 3 & 2,1 \\
\hline Licor/Pinga & 2 & 1,4 \\
\hline \multicolumn{3}{|l|}{ Doses consumidas } \\
\hline 1 a 2 doses & 65 & 44,6 \\
\hline 3 a 4 doses & 32 & 21,9 \\
\hline 5 a 6 doses & 21 & 14,4 \\
\hline 7 a 9 doses & 5 & 3,4 \\
\hline 10 ou mais doses & 6 & 4,1 \\
\hline \multicolumn{3}{|l|}{ Frequiência de embriaguez } \\
\hline 1 a 5 vezes & 26 & 17,8 \\
\hline 6 a 19 vezes & 5 & 3,4 \\
\hline 20 vezes ou mais & 3 & 2,1 \\
\hline \multicolumn{3}{|l|}{ Local de uso } \\
\hline Em casa & 13 & 8,9 \\
\hline Bares/danceterias/boates & 101 & 69,2 \\
\hline Casa de amigos & 20 & 13,7 \\
\hline \multicolumn{3}{|l|}{ Pessoas com as quais costumam beber } \\
\hline Familiares & 12 & 8,2 \\
\hline Amigos & 114 & 78,1 \\
\hline Outros & 1 & 0,7 \\
\hline
\end{tabular}

Tabela 6. Eventos ocorridos após beber em estudantes do curso de medicina do Centro de Ciências da Saúde da Universidade Federal do Espírito Santo, Vitória, 2007.

\begin{tabular}{lcc}
\hline \multicolumn{1}{c}{ Eventos ocorridos } & $\mathbf{n}$ & $\%$ \\
\hline Brigou & 8 & 5,5 \\
Sofreu acidentes & 9 & 6,2 \\
Dirigiu & 27 & 18,5 \\
Faltou à faculdade & 21 & 14,4 \\
Faltou ao trabalho & 0 & 0 \\
Nenhum & 27 & 18,5 \\
Não respondeu & 54 & 37 \\
Total & 146 & 100 \\
\hline
\end{tabular}

\section{DISCUSSÃO}

O número de sujeitos deste estudo foi de 168 e em sua maioria mulheres (54,8\%), na faixa etária entre 17 e 22 anos. Os achados do presente estudo divergiram do trabalho de Souza, Landim e Perdigão ${ }^{7}$, com acadêmicos de medicina no Ceará, em que a maioria (57\%) pertencia ao sexo masculino. Também pode-se perceber a dificuldade da inserção, por parte dos estudantes de níveis sociais mais baixos, na universidade, pois, neste estudo, a maioria pertence às classes sociais $A$ e $B$, que são as mais elevadas ( $A=40,5 \%$; $B=50 \%$ ), dado freqüentemente encontrado nos cursos de medicina. Resultado semelhante foi detectado por Lucas et al. ${ }^{19}$, em seu estudo com universitários da área da saúde da Universidade Federal do Amazonas, em que $56 \%$ dos estudantes pertenciam à classe $A$ e 27,3\% à classe B.
O trabalho mostra que os alunos de medicina da UFES não apresentam diferenças em relação ao consumo de álcool quando comparados com a população em geral. No presente estudo, a prevalência encontrada para uso de álcool na vida foi de $86,9 \%$, semelhante aos achados de Kerr-Correa et al. ${ }^{10}$, que encontrou 84\% de estudantes que já haviam feito uso de álcool na vida. Também não diferem dos estudos de Souza, Landim e Perdigão', que encontraram prevalência de 92\% para uso de álcool na vida. Em relação ao uso de álcool no ano foi detectado $82,1 \%$, resultado expressivo quando comparado ao achado de Silva et al. ${ }^{20}$, em que 64,1\% dos escolares de ensino médio do município de São José do Rio Preto, SP, já haviam feito uso de álcool nos últimos 12 meses. Deve-se levar em consideração, entretanto, a diferença de idade entre os sujeitos dos referidos estudos. Quanto ao uso de álcool no mês, identificou-se que 48,2\% dos universitários pesquisados situavam-se neste padrão, dado próximo ao encontrado por Alves et al. ${ }^{21}$, em que 53,2\% dos estudantes relataram o uso de álcool no último mês. $\bigcirc$ uso freqüente e o uso pesado de álcool encontrados neste estudo (13,7\% e 3\%, respectivamente) foram muito próximos aos descritos por Tavares et al.22, em sua pesquisa com estudantes adolescentes, em que 16,8\% faziam uso freqüente e $5 \%$ faziam uso pesado dessa substância. Neste sentido, observa-se que os usos freqüente e pesado do álcool revelam dados preocupantes, pois entre os adolescentes a ocorrência é um pouco maior que entre os universitários, chamando a atenção para que as políticas e programas de prevenção se destinem às faixas etárias mais baixas, não obstante, devam também responder à demanda advinda da classe universitária. O tabaco se apresenta como a segunda droga de maior uso na vida (22\%) e no ano (10,7\%). Resultados que corroboram os de Guimarães et al..$^{23}$ em relação ao uso na vida (22,7\%), mas difere dos achados de Souza, Landim e Perdigão em relação ao uso no ano (26,5\%). Quanto ao uso freqüente (1,8\%) e pesado $(0,6 \%)$, os resultados estão de acordo com os encontrados por Kerr-Correa et $a l^{10}{ }^{10}$ em que não houve uso freqüente e apenas $2 \%$ dos estudantes fizeram uso pesado. Este, por sua vez, mostrou-se bastante inferior ao descrito por Menezes et al. ${ }^{24}$, em seu estudo com 449 estudantes de medicina de Universidade Federal de Pelotas, em que 11,6\% dos sujeitos relataram usar tabaco com freqüência diária nos 30 dias anteriores. Em relação ao uso no mês, o resultado do presente estudo $(1,2 \%)$ foi menor que o encontrado por Kerr-Correa et al. ${ }^{10}$ (7\%).

Entre os medicamentos com potencial para abuso, os mais utilizados na vida foram os ansiolíticos (13,1\%) e as anfetaminas (10,1\%). De acordo com Pinton et al. ${ }^{25}$, os acadêmicos de medicina usam tais medicamentos em virtude do extenso conteúdo curricular a ser estudado, com o intuito de ter mais atenção e/ou ficar acordado durante mais tempo. Em seu estudo com universitários de medicina, o uso na vida de anfetaminas foi de 21,5\% e o de ansiolíticos de 13,4\%.

Em relação às drogas ilícitas, os solventes aparecem em primeiro lugar, com 15,5\% de uso na vida, sendo seguido da 
cannabis sativa $(9,5 \%)$ e dos alucinógenos (1,8\%). O mesmo ocorre entre os achados de Galduróz et al. ${ }^{9}$ no V Levantamento sobre o Consumo de Drogas entre Estudantes de Ensino Fundamental e Médio da Rede Pública, no qual os solventes aparecem em primeiro lugar (15,5\%), seguido da cannabis sativa $(5,9 \%)$ e da cocaína $(2,0 \%)$.

Fazendo a análise do uso de substâncias psicoativas em relação ao sexo, pode-se perceber que o consumo varia em função do gênero. O consumo de álcool variou pouco em função do sexo, uma vez que 89,2\% dos homens fizeram uso na vida de álcool contra $84,8 \%$ das mulheres. Já em relação ao tabaco, houve diferença maior entre os sexos, em que entre os homens, 25,7\% já fizeram uso e apenas 19,6\% das muIheres o fizeram. Em relação ao consumo de anfetamínicos, o sexo pouco influenciou, já que 10,8\% dos homens já fizeram uso contra 9,8\% das mulheres. Já no uso de cannabis sativa (13,5\% dos homens e 6,5\% das mulheres) e solventes (18,9\% dos homens e $13 \%$ das mulheres), observou-se diferença significativa no consumo entre os sexos. Quanto aos ansiolíticos, as mulheres apresentaram maior uso na vida $(16,3 \%)$ que os homens (9,5\%). Tavares et al. ${ }^{26}$ não encontrou diferenças entre os sexos em adolescentes escolares, só ocorrendo com o uso de álcool e anfetamínicos. E, para Silva et al.27, excetuando o álcool, todas as outras substâncias, entre os homens, tiveram maior prevalência. Já no trabalho de Tavares et al.22, foi encontrado o mesmo perfil de consumo em relação ao sexo, visto que somente o consumo de tabaco não apresentou diferenças significativas entre homens $(40,1 \%)$ e mulheres (43\%).

O consumo de substâncias psicoativas tem sido cada vez mais precoce. No presente estudo, as faixas etárias de maior prevalência para a experimentação das drogas são as de 13 a 15 anos, de 16 a 18 anos e acima de 18 anos. Entre as faixas etárias de experimentação, a de 13 a 15 anos foi bastante recorrente para o álcool (28,1\%), o tabaco $(16,2 \%)$ e os solventes $(15,4 \%)$. Contudo, mesmo entre estas drogas, é nas faixas etárias de 16 a 18 anos e acima de 18 anos que ocorre o maior número de experimentação das substâncias psicoativas. Nestas faixas etárias, 39,75\% dos estudantes já haviam utilizado o álcool; 43,25\%, o tabaco; $62,5 \%$, a cannabis sativa; $34,2 \%$, os solventes; $76,5 \%$, as anfetaminas e $54,5 \%$, os ansiolíticos. Estes resultados corroboram os dados de Lucas et al.19, em que todas as substâncias tiveram maior experimentação entre 16 a 18 anos ou acima dos 18 anos, com exceção do tabaco cuja faixa etária de maior experimentação foi entre 13 e 15 anos. A idade de experimentação pode estar diretamente influenciada pela inserção deste indivíduo na vida acadêmica, em que, muitas vezes, o estudante vive em repúblicas, onde adquire maior liberdade por estar longe dos pais, ou simplesmente pela maior responsabilidade advinda por causa da vida acadêmica, o que pode lhe conferir maior autonomia ${ }^{25}$.

Aprofundando um pouco mais a análise acerca do consumo de álcool, observou-se que as bebidas de preferência para os estudantes foram a cerveja $(50,4 \%)$ e a vodca $(15,1 \%)$. Apesar de a vodca figurar entre as bebidas mais consumidas, deve-se ressaltar as condições nas quais ela é ingerida, ou seja, sob a forma de coquetéis ou drinques, muito comuns nas festas organizadas por tais universitários, sendo pouco consumida sem que o usuário a dilua em outra substância. Pinton et al. ${ }^{25}$ também encontraram a cerveja ou o chope $(66,5 \%)$ em primeiro lugar na preferência dos universitários. Os estudos de Lucas et al. ${ }^{19}$ também confirmam a cerveja em primeiro lugar (31,8\%), mas apresentam o vinho como segunda bebida mais consumida $(13,7 \%)$.

Em relação aos locais onde os acadêmicos de medicina mais consomem álcool, os mais mencionados foram os bares, as boates e as danceterias $(69,2 \%)$ e $78,1 \%$ dos estudantes relataram beber na companhia de amigos. Os achados diferem um pouco dos resultados de Alves et al. ${ }^{21}$, em que $65 \%$ dos adolescentes das escolas públicas de Feira de Santana, BA, referiram as festas periódicas como o lugar preferido para consumir tais bebidas.

Além da freqüência com que se bebe, o que se bebe, onde e com quem se bebe, outro dado fundamental é saber quanto se consome em uma única ocasião. É na quantidade de doses tomadas em um único dia que o beber como lazer pode transformar-se em uso abusivo do álcool, com danos para a saúde que vão da exposição a doenças ao risco de acidentes graves ${ }^{3}$. No presente estudo descobriu-se que entre os estudantes da faculdade de medicina da UFES que relataram uso na vida de álcool, 21,9\% bebem 3 a 4 doses e 14,4\% bebem 5 a 6 doses. Dos que disseram consumir entre 7 e 9 doses por ocasião, que representaram 3,4\% e, ainda, 4,1\% daqueles estudantes que disseram consumir 10 ou mais doses por ocasião. Estes dados corroboram com os achados de Laranjeira et al. ${ }^{3}$, em seu Levantamento Nacional sobre os Padrões de Consumo do Álcool na População Brasileira, em que, dos indivíduos de 18 a 24 anos, 24\% bebem de 5 a 11 doses por ocasião.

Segundo Laranjeira et al. ${ }^{3}$, este comportamento de beber muitas doses em uma única ocasião está mais presente na faixa etária de 18 a 24 anos e 22\% destes bebem com tais características ao menos uma vez por semana. De acordo com Brewer et al., citado por Laranjeira et al. ${ }^{3}$, o consumo de 5 doses ou mais (homens), ou 4 doses ou mais (mulheres) em uma mesma ocasião caracteriza o beber em binge, termo internacionalmente utilizado para designar uso pesado episódico do álcool e que causa mais custos sociais e de saúde que o uso contínuo e dependente. Quando questionada acerca do número de doses consumidas por ocasião, nos últimos 12 meses antecedentes à entrevista, aproximadamente $28 \%$ da população brasileira afirmou que bebeu em binge ${ }^{3}$.

Este uso nocivo do álcool, dependendo do peso, da idade, da rapidez com que consome bebida alcoólica e ter se alimentado ou não, entre outros fatores, pode levar o indivíduo 
à embriaguez ${ }^{3}$. Entre os estudantes de medicina da UFES que relataram uso na vida de álcool, 17,8\% disseram ter chegado a um estado de embriaguez de 1 a 5 vezes nos últimos 30 dias, $3,4 \%$ confessaram ter ficado embriagados de 6 a 19 vezes e 2,1\% de 20 dias ou mais nos últimos 30 dias. Estes resultados foram um pouco maiores do que aqueles encontrados por Lucas et al. ${ }^{19}$, em que 6,1\% relataram embriaguez de 1 a 5 dias nos últimos 30 dias. Contudo, na freqüência de embriaguez de 6 a 19 dias e, na freqüência de embriaguez em 20 dias ou mais, os percentuais foram semelhantes, com $4 \%$ e $2,9 \%$, respectivamente.

O consumo de álcool, além de trazer prejuízos para o próprio consumidor, levanta também questões de cunho social, pois, muitas vezes, afeta não só aquele que consome, mas, também, as pessoas que o cercam. Neste estudo, 18,5\% dos estudantes que consumiram bebidas alcoólicas relataram já ter dirigido após beber; $14,4 \%$ disseram ter faltado às aulas, 6,5\% confessaram ter sofrido algum acidente e 5,5\% se envolveram em brigas. Estes resultados vêm confirmar o exposto por Pinton et al. ${ }^{25}$, em que $60 \%$ dos alunos pesquisados faltaram às aulas após beber, 44,7\% dirigiram e 15,4\% se envolveram em brigas. Também Lucas et al. ${ }^{19}$ descrevem que $47,3 \%$ dos estudantes relataram ter dirigido após beber, $33,7 \%$ faltaram às aulas, $4,7 \%$ se envolveram em brigas e 2,4\% se envolveram em algum tipo de acidente.

\section{CONCLUSÃO}

O perfil do uso das substâncias psicoativas por acadêmicos de medicina da UFES revelou que é preocupante o alto consumo de drogas lícitas, com especial atenção para o álcool, que por características socioculturais é muito subestimado. Também o tabaco, responsável pelo aumento da incidência de diversos cânceres, as anfetaminas e ansiolíticos, além das drogas ilícitas (com ênfase para os solventes), aparecem nos resultados do presente estudo, levantando questões acerca da falta de maturidade destes alunos, futuros profissionais da saúde, em relação aos riscos envolvidos no uso de SPAs e em relação a todas as conseqüências pessoais, sociais e econômicas advindas do uso abusivo de substâncias psicoativas.

Fica clara, então, tendo em vista a melhor formação não só de um profissional de saúde, mas de um ser humano mais saudável, a necessidade da inserção de disciplinas curriculares no curso de medicina da UFES, que tratem do tema álcool e outras drogas, bem como do tema saúde mental e assistência ao paciente farmaco-dependente. Faz-se necessária, também, a criação de programa dentro do CCS da UFES, voltado para a prevenção do uso de drogas entre os universitários, pois, uma vez cientes do perfil da população de risco a ser assistida, a prevenção pode ser desenvolvida de maneira muito mais efetiva.

\section{AGRADECIMENTOS}

Ao Núcleo de Estudos sobre Álcool e outras Drogas (NEAD) pela oportunidade de realizar a pesquisa. À Fundação de Apoio à Pesquisa do Espírito Santo (Fapes) pelo suporte financeiro. À Paula Silva Mardegan, Flávia Batista Portugal e Renata Frossard Teixeira pelo auxílio na coleta de dados.

\section{REFERÊNCIAS}

1. Smart RG, Hugues PH, Johnston LD, Anumonye A, Khant U, Mora MEM, et al. A methodology for students drug-use surveys. Geneva: World Healh Organization; 1980.

2. Statistical Package Social Science (SPSS) for windows. Versão 2005. Chicago: SPSS Inc:; 2005.

3. Laranjeira R, Pinsky I, Zalesky M, Caetano R. I Levantamento Nacional sobre Padrões do Consumo de Álcool na População Brasileira. Brasília, 2007.

4. Brasil. Presidência da República. Secretaria Nacional Antidrogas. Política Nacional sobre Drogas. Braślia: Presidência da República, Gabinete de Segurança Institucional, Secretaria Nacional Antidrogas, 2005.

5. Carlini EA, Galduróz JCF, Silva AAB, Noto AR, Fonseca AM, Carlini CM, et al. II Levantamento Domiciliar sobre o Uso de Drogas Psicotrópicas no Brasil: estudo envolvendo as 108 maiores cidades do país, 2004. São Paulo: Centro Brasileiro de Informações sobre Drogas Psicotrópicas (Cebrid). Departamento de Psicobiologia da Escola Paulista de Medicina, 2005.

6. Hughes $H$, et al. Prevalence of substance use among physicians. JAMA. 1992;267:2333-9.

7. Souza FGM, Landim RM, Perdigão FB. Consumo de drogas e desempenho acadêmico entre estudantes de medicina no Ceará. Rev Psiq Clin. 1997;26(4):188-99.

8. Boskovitz EP, Cruz ETN, Chiaravalotti-Neto F. Uso de drogas em universitários em São José do Rio Preto, São Paulo. Rev Psiquiatr Clin. 1995; 22:87-93.

9. Galduróz JCF, Noto AR, Fonseca AM, Carlini EA. V levantamento nacional sobre o consumo de drogas psicotrópicas entre estudantes do ensino fundamental e médio da rede pública de ensino nas 27 capitais brasileiras, 2004. São Paulo: Centro Brasileiro de Informações sobre Drogas Psicotrópicas (CEBRID). Departamento de Psicobiologia da Escola Paulista de Medicina, 2004

10. Kerr-Correa F, Andrade AG, Bassit AZ, Boccuto MNVF, et al. Alcohol and drug use by Unesp medical students. Rev Bras Psiquiatr. 1999;21(2).

11. Turato RE. Tratado de metodologia da pesquisa clínico-qualitativa. Petrópolis: Vozes; 2003.

12. Woodward M. Epidemiology study design and data analysis. Chap man Hall/CRC; 1999.

13. Andrade AG, Bassit AZ, Mesquita AM, Fukushima JT, Gongalves EL. Prevalência do uso de drogas entre alunos da Faculdade de Medicina da Universidade de São Paulo (1991-1993). Rev ABP-APAL. 1995;17:41-6.

14. Mesquita AMC, Bucaretchi HA, Castel S. Estudantes da faculdade de medicina da Universidade de SP: uso de SPAs em 1991. Revista ABP-APM. 1995;17(2):47-54.

15. Carlini-Cotrim B. 0 uso de drogas psicotrópicas por estudantes do primeiro e segundo graus da rede estadual, em dez capitais brasileiras, 1987. In: Consumo de drogas psicotrópicas no Brasil, em 1987. Centro de Documentação do Ministério da Saúde, 1989. p. 84-9.

16. Conselho Nacional de Saúde (CNS). Resolução n. 196/96 regulamenta e estabelece as diretrizes para as pesquisas envolvendo seres humanos no Brasil. 1996. [acesso em 2006 Ago 10]. Disponível em: http://www.conselho.saude.gov.br/ [2005 14 mar].htm.

17. Associação Brasileira dos Institutos de Pesquisa de Mercado (Abipeme). Proposição para um novo critério de classificação socioeconômica, 1978. Mimeo. São Paulo, 1978. 15p.

18. Carlini-Cotrim B, Barbosa MTS. Pesquisas Epidemiológicas sobre o uso de drogas por estudantes: um manual de orientações gerais. São Paulo: Cebrid; 1993.

19. Lucas ACS, Parente RCP, Picanço NS, Conceição DA, Costa KRC, Magalhães IRS, et al. Uso de psicotrópicos entre universitários da área da saúde da Universidade Federal do Amazonas, Brasil. Cad Saude Publica. 2006;22(3):663-71. 
20. Silva EF, Pavani RAB, Moraes MS, Chiaravalotti Neto F. Prevalência do uso de drogas entre escolares do ensino médio do Município de São José do Rio Preto, São Paulo, Brasil. Cad Saude Publica. 2006;22(6):1151-8.

21. Alves MVQM, Costa COM, Nascimento Sobrinho CL, Santos CAST, Gomes WA, Assis DR. Uso de bebidas alcoólicas entre adolescentes: perfil de experimentação, uso regular e fatores de risco. Rev Baiana Saude Publica. 2005;29(1):91-104.

22. Tavares BF, Béria JU, Lima MS. Prevalência do uso de drogas e desempenho escolar entre estudantes. Rev Saude Publica. 2001;35(2):150-8.

23. Guimarães JL, Godinho PH, Cruz R, Kappann Jl, Tosta Junior LA. Consumo de drogas psicoativas por adolescentes escolares de Assis, SP. Rev Saude Publica. 2004;38(1):130-2.
24. Menezes A, Palma E, Holthausen R, Oliveira R, Oliveira OS, Devens E, et al. Evolução temporal do tabagismo em estudantes de medicina, 1986, 1991, 1996. Rev Saude Publica. 2001;35(2):165-9.

25. Pinton FA, Boskovitz EP, Cabrera EMS. Uso de drogas entre os estudantes de medicina de São José do Rio Preto, SP, no ano de 2002. Arq Ciência Saúde. 2005;12(2):91-6.

26. Tavares BF, Béria JU, Lima MS. Fatores associados ao uso de drogas entre adolescentes escolares. Rev Saude Publica. 2004;38(6):787-96.

27. Silva LVER, Malbergier A, Stempliuk VA, Andrade AG. Fatores associados ao consumo de álcool e drogas entre estudantes universitários. Rev Saude Publica. 2006;40(2):280-8 